14.03

\title{
Математическое моделирование нарушений перфузии миокарда, диагностируемых методом однофотонной эмиссионной компьютерной томографии
}

\author{
(с) И.П. Колинко, ${ }^{1,2}$ Н.В. Денисова, ${ }^{1}$ А.А. Аншелес ${ }^{3}$ \\ ${ }^{1}$ Институт теоретической и прикладной механики им. С.А. Христиановича, \\ 630090 Новосибирск, Россия \\ ${ }^{2}$ Новосибирский государственный университет, \\ 630090 Новосибирск, Россия \\ ${ }^{3}$ Национальный медицинский исследовательский центр кардиологии им. Мясникова, \\ 121552 Москва, Россия \\ e-mail: i.kolinko@g.nsu.ru
}

Поступило в Редакцию15 декабря 2019 г.

В окончательной редакции 14 марта 2020 г.

Принято к публикации 16 марта 2020 г.

\begin{abstract}
Представлены результаты исследований, направленных на развитие представления математической модели миокарда левого желудочка сердца в режиме полярной карты „bulleye“ (бычий глаз). Сравнение изображений, полученных в результате численного моделирования, с подобными случаями в клинической практике позволило оценить возможные ошибки и неточности реконструкции в методе однофотонной эмиссионной компьютерной томографии.
\end{abstract}

Ключевые слова: ядерная медицина, математическое моделирование, Однофотонная эмиссионная компьютерная томография, ишемическое поражение сердца.

DOI: 10.21883/JTF.2020.09.49681.401-19

\section{Введение}

Однофотонная эмиссионная компьютерная томография (ОЭКТ) является современным методом визуализации и оценки кровоснабжения (перфузии) миокарда на клеточном уровне [1]. Этот метод основан на использовании радиофармацевтических препаратов (РФП), которые активно поглощаются здоровыми мышечными клетками сердца - кардиомиоцитами. В качестве радиоактивного индикатора в ядерной кардиологии наиболее частое применение имеет препарат метокси-изобутил-изонитрил (МИБИ), меченый технецием Тc99m ( $\left.{ }^{99 m} \mathrm{Tc}-\mathrm{MIBI}\right)$. Метод ОЭКТ с использованием ${ }^{99 m} \mathrm{Tc}-\mathrm{MIBI}$ позволяет проводить диагностику наличия, локализации, распространенности и тяжести ишемического поражения сердца, распространенности рубцовых постинфарктных изменений, а также количества жизнеспособного миокарда левого желудочка (ЛЖ) сердца. Несмотря на то что метод ОЭКТ широко используется в диагностике сердечно-сосудистых заболеваний, остались нерешенные проблемы, которые могут приводить к ошибкам на реконструированных изображениях и допускать неоднозначную трактовку полученных изображений [2]. Клинические методы исследований ограничены в изучении причин наблюдаемых неоднозначных результатов и возникающих ошибок. Чтобы оценить качество реконструированного изображения, необходимо сравнить его с „первоисточником“, т. е. действительным распределением РФП в органах па- циента, что абсолютно невозможно. Поэтому актуальной задачей в области диагностической ядерной медицины является развитие подхода на основе метода математического моделирования $[3,4]$. Этот подход включает в себя математическую модель распределения РФП в биологических тканях пациента (виртуальный пациент), математическую модель системы сбора сырых данных (виртуальный томограф), а также математическую программу создания реконструированного изображения распределения РФП по виртуальным данным. В численных экспериментах реконструированное изображение можно сравнить с распределением РФП в соответствующих органах виртуального пациента и оценить его точность, определить ошибки реконструкции и исследовать причины возникновения этих ошибок. Результаты численного моделирования должны быть представлены графически в том же виде, как они представляются при клинических обследованиях пациентов в соответствии с требованиями протокола. Это позволяет выполнить сравнение изображений, полученных в численном моделировании, с подобными случаями в клинической практике и оценить возможные ошибки и неточности реконструкции.

Современный протокол исследования перфузии миокарда методом ОЭКТ рекомендует оценивать распределение РФП в миокарде ЛЖ в режиме томографических срезов и полярных карт [5]. В настоящей работе развита математическая модель для представления миокарда ЛЖ сердца в режиме полярной карты „bulleye“ (бычий глаз). 


\section{1. Математическая модель распределения РФП в органах грудной клетки („виртуальный пациент“)}

Процедура обследования методом ОЭКТ начинается с внутривенного введения пациенту радиофармацевтического препарата. Как сказано выше (см. Введение), при исследовании перфузии (кровоснабжения) миокарда широко применяется МИБИ. МИБИ поступает в области миокарда с ненарушенным кровоснабжением и накапливается в здоровых клетках миокарда. Радионуклид ${ }^{99 m} \mathrm{Tc}$ является „меткой“, испускающей гамма-кванты с энергией $140 \mathrm{keV}$. Первоначально ${ }^{99 m} \mathrm{Tc}-$ МИБИ накапливается не только в миокарде, но также в печени и желудочнокишечном тракте (ЖКТ). Поскольку у многих пациентов левая доля печени непосредственно примыкает к нижней стенке сердца, это может затруднить интерпретацию перфузии нижней стенки ЛЖ. Поэтому выдерживается некоторый промежуток времени (примерно $40 \mathrm{~min}$ ) для клиренса РФП из печени и ЖКТ, и затем пациент помещается на стол томографической системы для детектирования выходящего гамма-излучения. Предполагается, что за время сбора данных на томографе распределение РФП в органах остается практически постоянным, при этом пациент не должен двигаться (за исключением жизненно необходимых движений, связанных с дыханием и биением сердца).

Математическая модель („виртуальный пациент“) описывает стационарное установившееся распределения РФП в органах грудной клетки. В работе [3] было представлено развитие „Математической Модели Торса“ (ММТ), описывающей анатомическое строение в области грудной клетки среднестатистического пациента мужского пола. Разработка фантома осуществлялась на основе уравнений аналитической геометрии и булевых логических операторов. На рис. 1 представлена полная анатомическая модель ММТ в стандартном положении пациента с поднятыми вверх руками. Модель включает органы грудной клетки, а также печень и петли кишечника. Следует отметить, что для моделирования процедуры
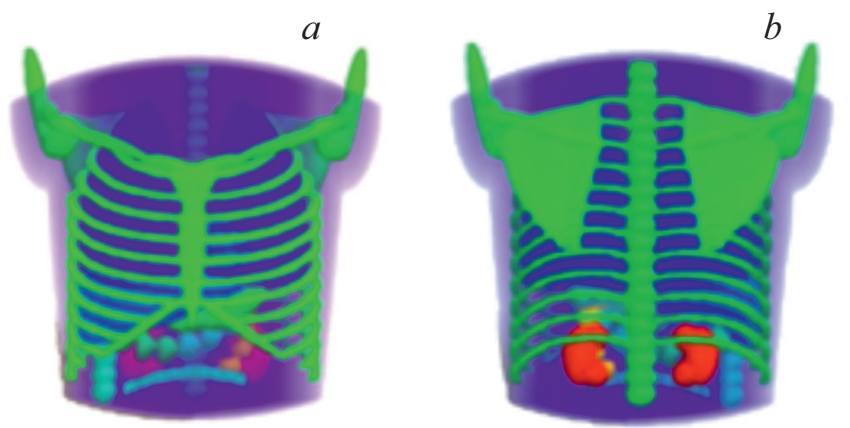

Рис. 1. $3 D$-анатомическая модель ММТ в стандартном положении пациента с поднятыми вверх руками: $a$ - вид модели ММТ спереди, $b$ - вид модели сзади.

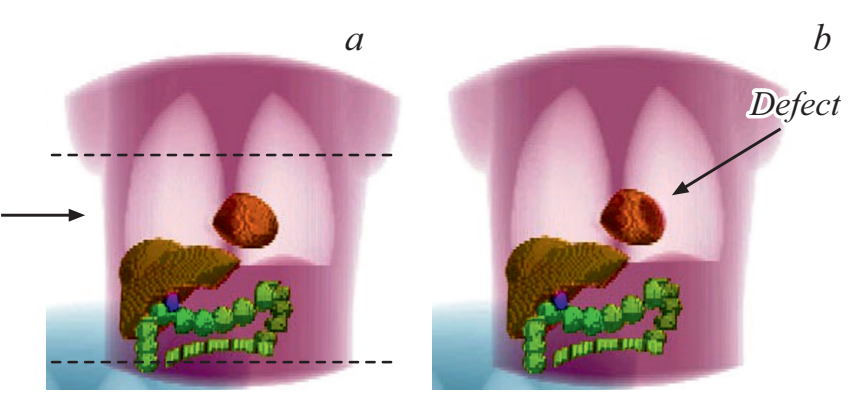

Рис. 2. 3D-эмиссионная модель ММТ-Э, описывающая распределение радиофармпрепарата ${ }^{99 m}$ Tc-MIBI в органах грудной клетки: $a$ - здоровый миокард, $b$ - миокард с ишемическим поражением. Штриховые линии на рис. 2, $a$ отмечают ту часть торса, которая попадает в поле зрения вращающейся гамма-камеры (обычно используются две гамма-камеры).

Относительное распределение концентрации РФП в различных органах

\begin{tabular}{l|c}
\hline \multicolumn{1}{c|}{ Орган } & Относительная концентрация РФП \\
\hline Торс (мягкие ткани) & 2 \\
Легкие & 1 \\
Печень & 40 \\
Желчный пузырь & 300 \\
Миокард & 40 \\
Петли кишечника & $20-30$
\end{tabular}

обследования перфузии миокарда фантом должен описывать именно распределение РФП, а не анатомическое строение пациента. На рис. 2 представлена „эмиссионная модель“ ММТ-Э, созданная на основе модели ММТ и описывающая распределения препарата $99 \mathrm{~m}$ Тс-МИБИ. В ядерной медицине эмиссионная модель далеко не всегда соответствует анатомическим структурам. Обычно на клинических планарных ОЭКТ изображениях желудок, поджелудочная железа, селезенка и почки не вносят значимого вклада, поэтому эти органы не представлены в модели ММТ-Э. Математическая модель MМТ развита в дискретном представлении. Для этого заданное $3 D$-пространство реконструкции $(R)$ дискретизировалось на отдельные воксели по схеме $64 \times 64 \times 64$ или $128 \times 128 \times 128$, и при этом каждый воксель идентифицировался номером $j$. В результате распределение РФП в организме представляется трехмерной матрицей чисел $n_{j}$, где $n_{j}$ соответствует концентрации препарата в вокселе с номером $j, j=1,2,3, \ldots, J$, $j \in R$.

При математическом моделировании первоначально задавалась относительная величина концентрации препарата ${ }^{99 m} \mathrm{Tc}-\mathrm{MIBI}$ в единице объема в соответствии с оценками клинических изображений. Затем эта величина пересчитывалась на основе известного значения полного числа гамма-квантов, зафиксированных гамма-камерами. 


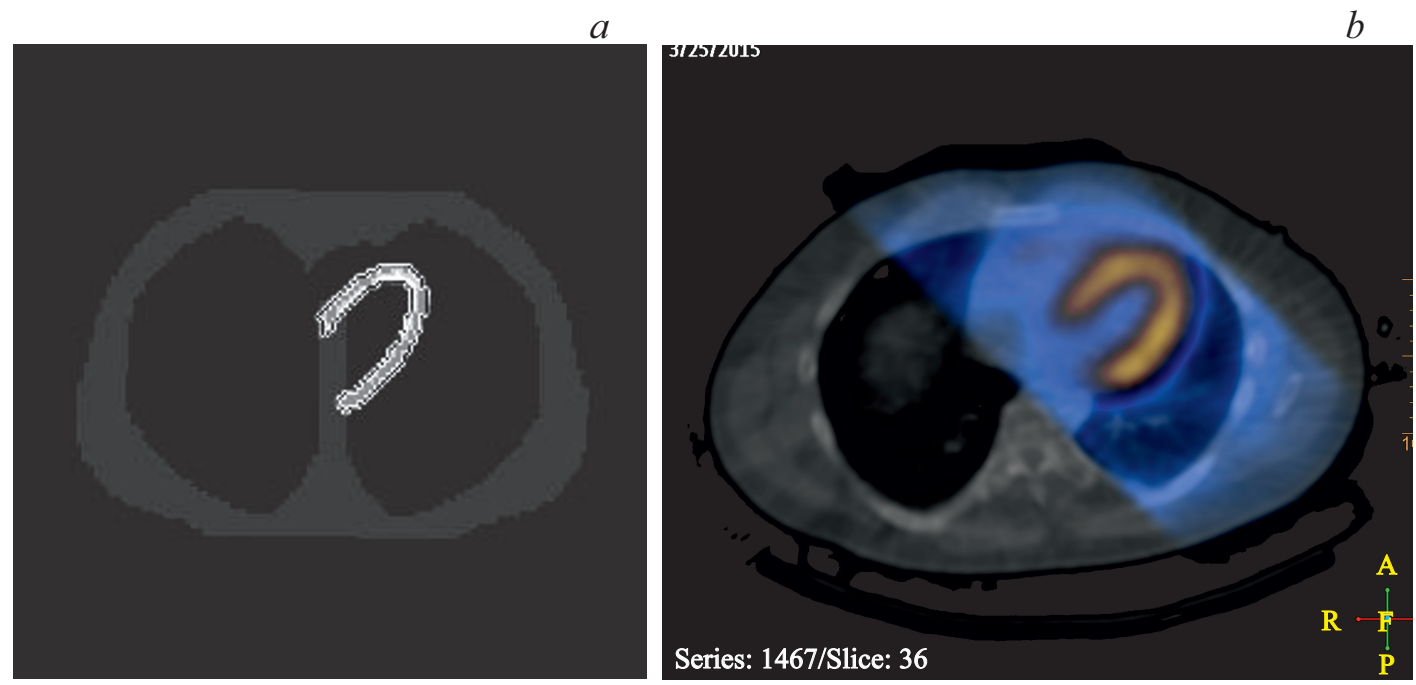

Рис. 3. Сравнение поперечного сечения модели ММТ-Э $(a)$ и поперечного сечения клинического ОЭКТ/КТ изображения $(b)$. Положение плоскости сечения модели ММТ-Э указано стрелкой на рис. $2, a$. Клиническое изображение получено в отделе РНД НМИЦ кардиологии наложением ОЭКТ и КТ изображений с помощью системы Philips Bright View XCT.

Использованные в модели (рис. 2) параметры представлены в таблице.

На практике значения этих концентраций могут сильно отличаться у разных пациентов в зависимости от интенсивности соответствующих метаболических процессов. Штриховые линии на рис. 2 отмечают ту часть торса, которая попадает в поле зрения вращающихся детекторов эмиссионного томографа.

При обследовании перфузии миокарда областью интереса является сердце. Поэтому важно, чтобы в развитой модели положение и ориентация сердца соответствовали клиническим данным. На рис. 3 представлено сравнение модели ММТ-Э и клинического ОЭКТ изображения в выделенной плоскости поперечного сечения. Положение плоскости указано стрелкой на рис. 2. Можно видеть, что локализация и ориентация сердца на модели соответствует клиническому ОЭКТ изображению, полученному в отделе радионуклидной диагностики НМИЦ кардиологии.

\section{2. Математическая модель распределения РФП в миокарде левого желудочка}

Как было отмечено выше, при обследовании перфузии миокарда областью интереса является сердце, поэтому при клинических ОЭКТ исследованиях изображение сердца выделяют из общей реконструированной картинки части торса, попавшей в поле зрения гамма-камеры. В современной ядерной кардиологии для визуальной оценки распределения РФП используется представление ЛЖ сердца в режиме томографических срезов: по вертикальной длинной оси (Vertical Long Axis, VLA), горизонтальной длинной оси (Horizontal
Long Axis, HLA) и короткой оси (Short Axis, SAX), a также в режиме полярной карты. Преобразование трехмерного изображения перфузии миокарда в полярные карты значительно облегчает интерпретацию и позволяет выполнить полуколичественные оценки и стандартизировать эти оценки для определения степени тяжести поражений ЛЖ сердца путем сравнения с базой данных (БД) здоровых пациентов. В качестве примера на рис. 4 представлены клинические результаты ОЭКТ обследования пациента, полученные в отделе РНД НМИЦ кардиологии. Показана реконструированная $3 D$-форма ЛЖ, его вертикальные и горизонтальные срезы по длинной оси, срезы по короткой оси и полярные карты. Для сравнения на рис. 5 представлено $3 D$ изображение миокарда левого желудочка, выделенное из модели ММТ-Э на рис. 2 и увеличенное. Также на рис. 5 показаны примеры изображений модели Лж в режиме томографических срезов. Следует отметить, что в норме на ОЭКТ изображениях практически не виден правый желудочек сердца и предсердия, поэтому на представленном рисунке изображен ЛЖ, что отличает ОЭКТ изображения от стандартных анатомических изображений сердца. В настоящей работе для моделирования формы и контура ЛЖ рассматривалась конечнодиастолическая фаза сердечного ритма. Математическая модель ЛЖ представлялась на основе двух вложенных друг в друга усеченных эллипсоидов. Сдвигая внутренний эллипсоид относительно его центра, можно получать модель ЛЖ с „апикальным утончением“. Размер и толщина стенок миокарда задавались на основе анализа данных, полученных при КТ (компьютерная томография) и ОЭКТ диагностике пациентов в отделе РНД НМИЦ кардиологии. 


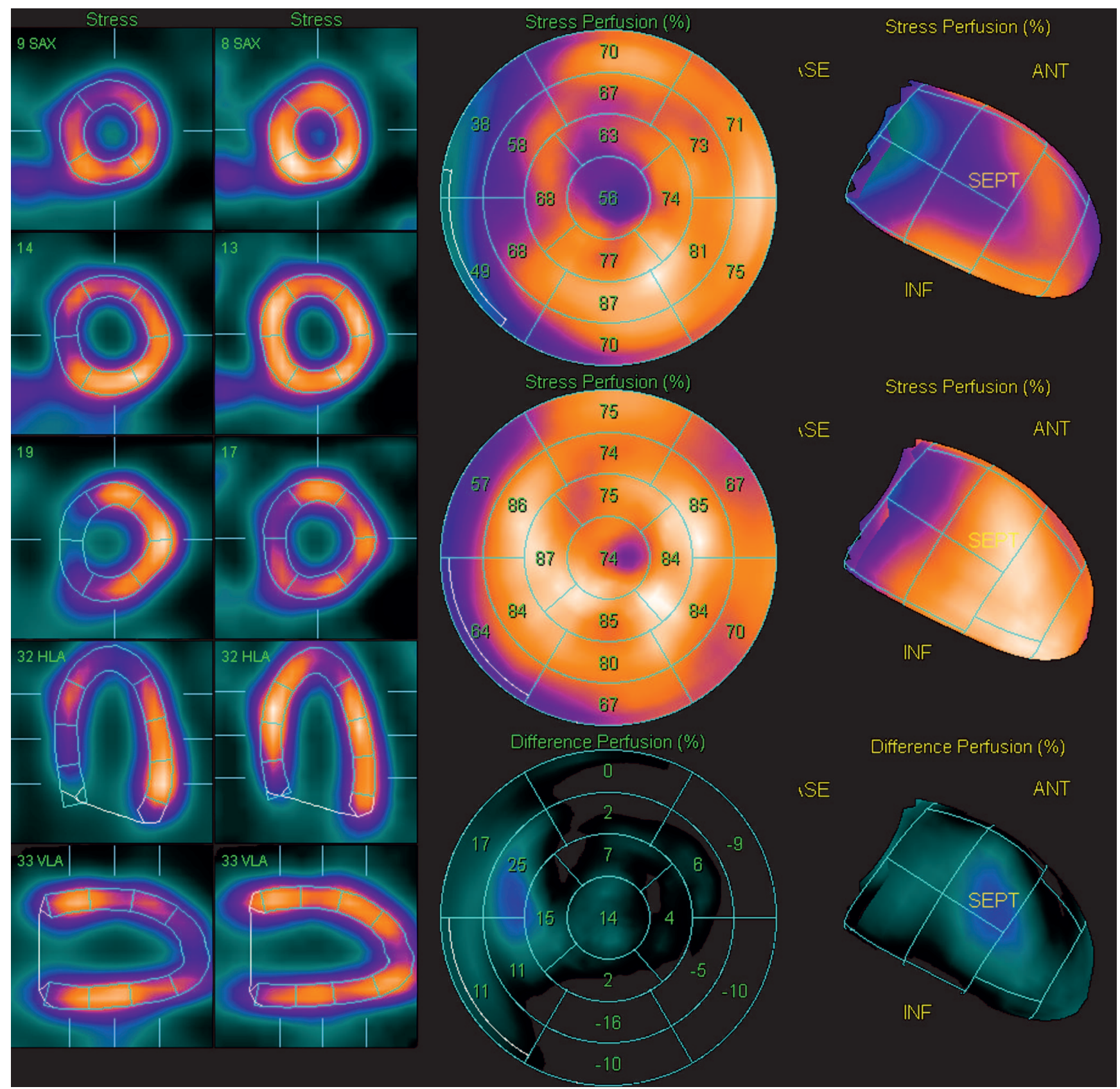

Рис. 4. Современное графическое представление результатов клинического ОЭКТ обследования перфузии миокарда. В первых двух столбцах представлены изображения ЛЖ в режиме томографических срезов: по вертикальной длинной оси (Vertical Long Axis, VLA), горизонтальной длинной оси (Horizontal Long Axis, HLA) и короткой оси (Short Axis, SAX). B третьем столбце представлены изображения ЛЖ в режиме полярных карт. В последнем столбце продемонстрированы $3 D$-изображения миокарда ЛЖ.

\section{3. Математическая модель представления миокарда лЖ сердца в режиме полярной карты „bull eye“ (бычий глаз)}

Как видно из рис. 5, представление ЛЖ в режиме срезов позволяет диагностировать наличие и локализацию поражения. Однако более наглядным и удобным явля- ется представление миокарда ЛЖ в режиме полярной карты, которое в англоязычной литературе получило название „bull еуе“ - „бычий глаз“. Примеры подобных карт представлены в третьей колонке на рис. 4. Можно видеть, что миокард ЛЖ разделен на клинических изображениях на 4 круговые зоны, и каждая зона разделена на определенное количество секторов. Программы, осуществляющие представление ЛЖ в полярном режиме, являются коммерческой собственностью компаний- 


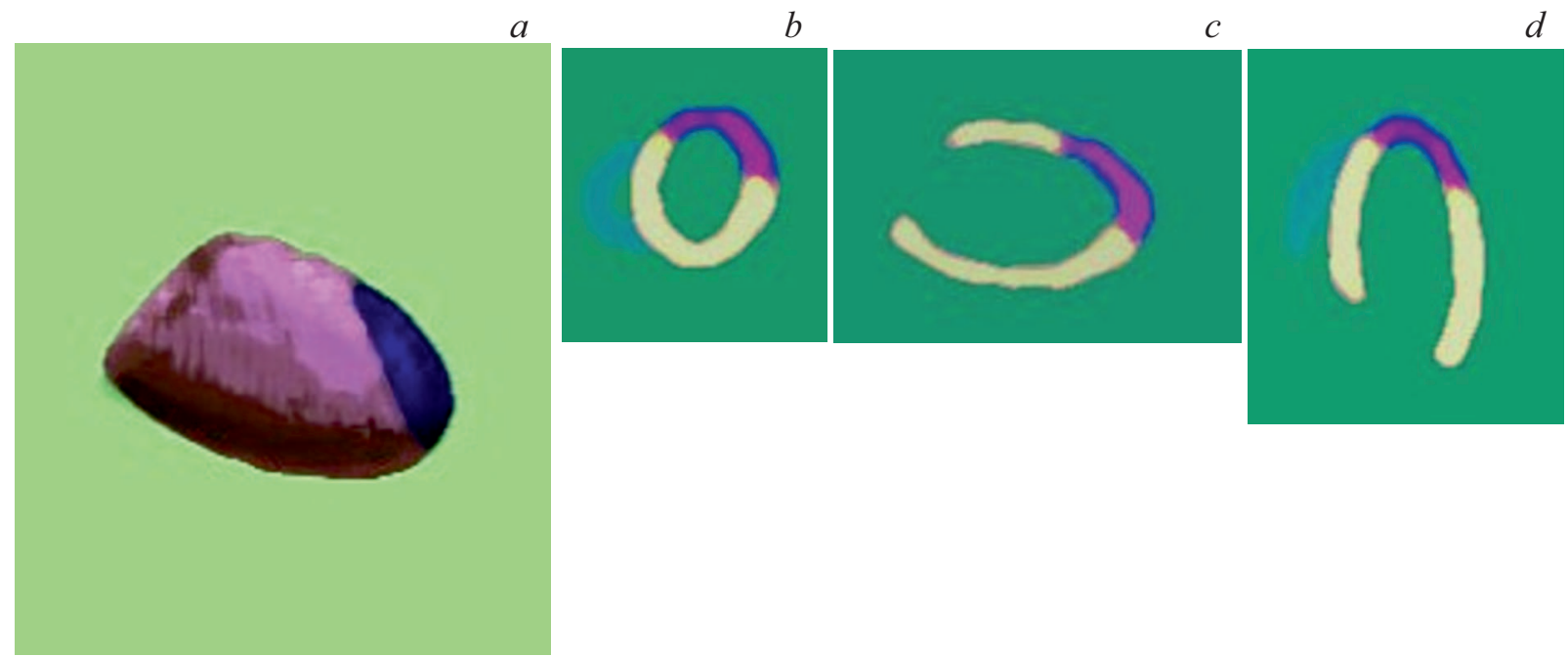

Рис. 5. Представление модели миокарда ЛЖ с дефектом в передне-апикальной зоне в режиме томографических срезов: $a-$ $3 D$-изображение модели миокарда ЛЖ, выделенное из рис. $2, b$ и увеличенное, $b$ - срез по короткой оси (Short Axis, SAX), $c$ - срез по вертикальной длинной оси (Vertical Long Axis, VLA), $d-$ срез по горизонтальной длинной оси (Horizontal Long Axis, HLA).

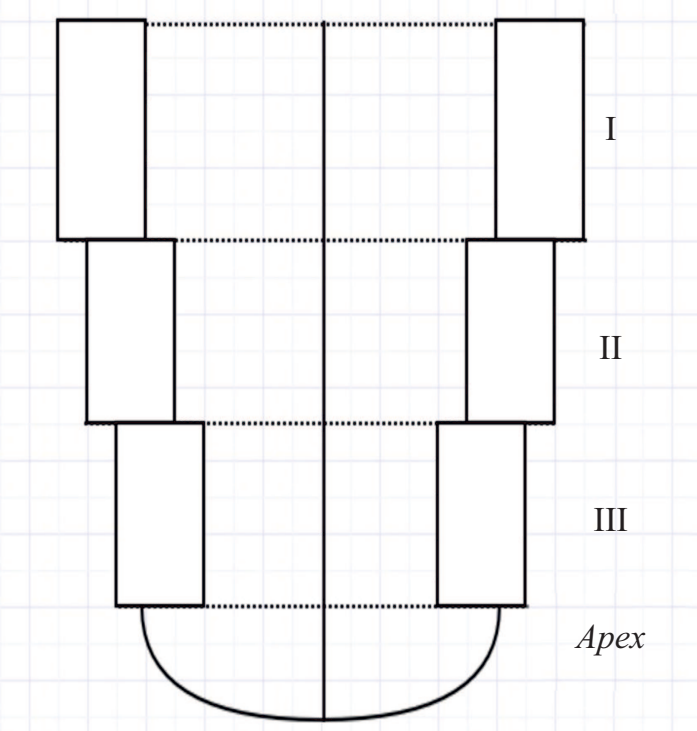

Рис. 6. $3 D$-модель ЛЖ, поделенная на 4 зоны: I - базальная часть миокарда ЛЖ, II - средняя часть, III - апикальная часть ЛЖ, IV - апекс (верхушка).

производителей оборудования. В численном моделировании различных клинических случаев для оценки погрешностей восстановления площади поражения и его значимости необходимо представлять результаты реконструкции в соответствии с принятыми клиническими нормами. Поэтому в настоящей работе выполнено математическое моделирование представления модели ЛЖ в полярной диаграмме.

$3 D$-модель ЛЖ представлена в замкнутой области в декартовой системе координат. Область разбита на вокселей одинакового размера. $3 D$-модель ЛЖ была поделена на 4 зоны, как это показано на рис. 6. Зона I соответствует базальной части миокарда ЛЖ, зона II средней части, зона III - апикальная часть ЛЖ и зона IV - апекс (верхушка). При моделировании полярной карты зона апекса рассматривалась отдельно, так как она имеет отличную от остальных частей ЛЖ геометрию. Общий принцип построения полярной диаграммы рассмотрим на примере зоны I. Вначале рассчитываем число воксельных слоев, попадающих в эту зону по вертикальной оси (рис. 6). Пусть число слоев равно $K$. В настоящей работе толщина стенки ЛЖ была практически равна линейному размеру вокселя. Каждый слой соответствует кольцу на полярной диаграмме. Таким образом, получили $K$ концентрических колец, причем верхний слой вокселей из зоны I соответствует внешнему кольцу полярной диаграммы, а нижний слой вокселей отображается на кольцо, расположенное ближе к центру. Для каждого из $K$-колец определялся размер единичного углового сегмента, соответствующий одному вокселю, и в текущем угловом сегменте задавалось значение активности из соответствующего вокселя $3 D$-модели. Обходим каждое из колец по кругу по часовой стрелке (с соответствующим угловым шагом). Записываем значения концентраций, полученные на пересечении каждого луча и кольца в отдельный массив. Эти данные затем используем для проекции каждого из колец на плоскость. Развитый подход к созданию изображений ЛЖ миокарда в режиме полярных карт был протестирован в численных экспериментах. В первом эксперименте задавалась $3 D$-модель здорового миокарда. Здоровый миокард имеет равномерное распределение РФП. На рис. 7 эта модель представлена в режиме томографических срезов и режиме полярной 


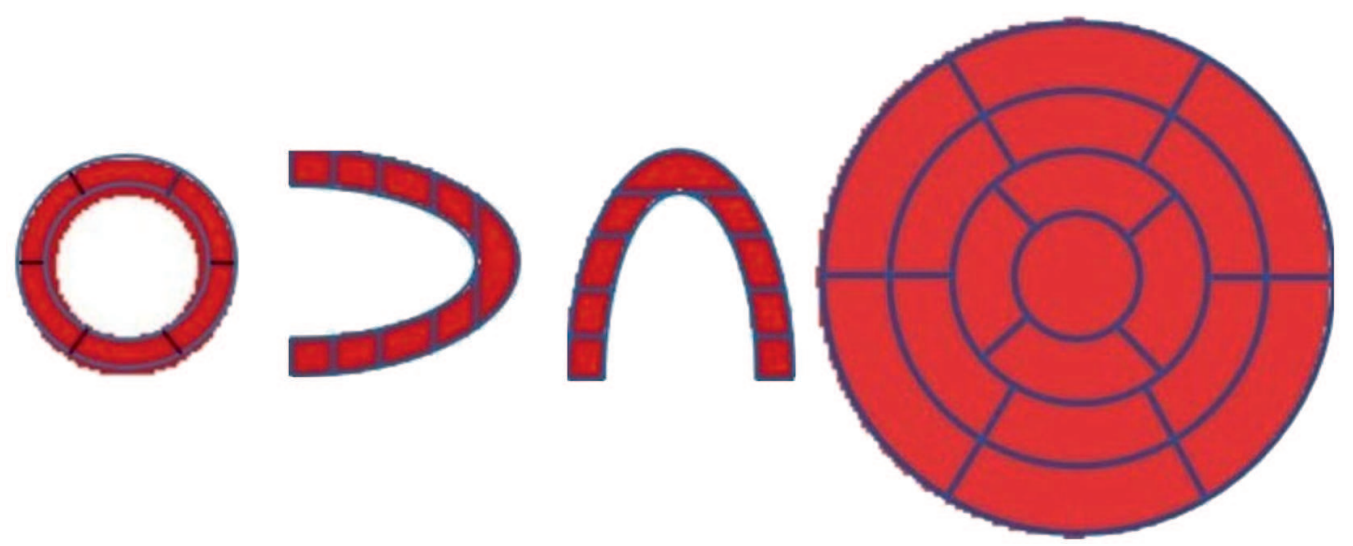

Рис. 7. Модель здорового ЛЖ сердца: - изображение здорового ЛЖ в режиме томографических срезов, $b-$ полярная диаграмма „bulleye“ здорового ЛЖ.
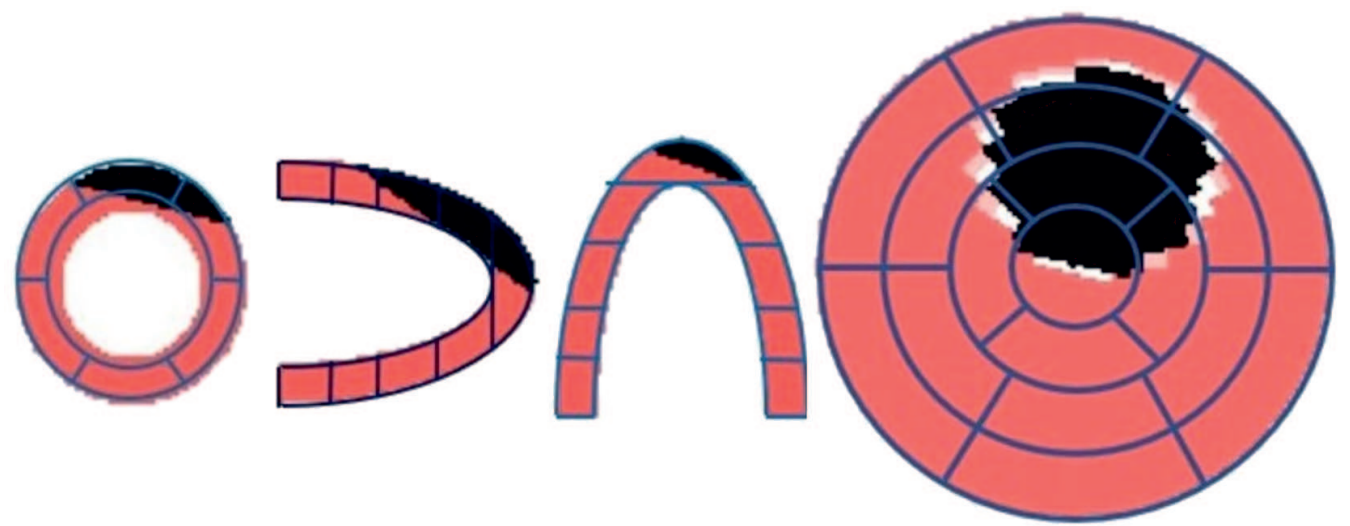

Рис. 8. Модель ЛЖ сердца с дефектом, имитирующим ишемическое поражение: $a$ - изображения ЛЖ сердца с дефектом в режиме томографических срезов, $b$ - полярная диаграмма „bulleye“ ЛЖ сердца с дефектом.

карты. Во втором эксперименте задавалась $3 D$-модель ЛЖ с дефектом, моделирующим ишемическое поражение. На рис. 8 представлено изображение этой модели в режиме срезов и выполнена трансформация в полярную диаграмму. Как видно из рис. $8, b$, представление в виде полярной диаграммы позволяет наглядно оценить площадь поражения.

\section{Заключение}

Представлены первые результаты исследований, направленных на изображение миокарда ЛЖ сердца в режиме полярной карты „,bulleye“ (бычий глаз). В работе толщина стенки ЛЖ была практически равна линейному размеру вокселя. В дальнейшем этот подход будет развит и для более сложного случая, когда толщина стенки включает несколько линейных размеров вокселя. Предполагается дальнейшее развитие этого подхода для количественных оценок значимости поражений, сравнения реконструированных изображений в режиме полярной карты с „точной“ моделью. Это позволит исследовать потенциальные ошибки в оценке площади поражения ЛЖ при обследовании перфузии миокарда методом ОЭКТ.

\section{Финансирование}

Исследования выполнены при поддержке гранта РФФИ № 19-02-00244-а.

\section{Конфликт интересов}

Авторы заявляют, что у них нет конфликта интересов.

\section{Список литературы}

[1] Jing Wu, Chi Liu // Phys. Med. Biol. 2019. Vol. 64. TR01. P. 37.

[2] Аншелес А.А. // Вестник рентгенологии и радиологии. 2014. № 2. C. 5-20.

[3] Denisova N.V., Terekhov I.N. // Biomed. Phys. Eng. Express. 2016. Vol. 2. N 5. P. 055015(12). DOI: $10.1088 / 2057-1976 / 2 / 5 / 055015$

[4] Денисова Н.В. // ЖТФ. 2018. Т. 88. Вып. 9. С. 1418-1426.

[5] Lin G.S., Hines H.H., Grant G., Taylor K., Ryals C. // Nucl. Med. Technol. 2006. Vol. 34. P. 3-17. 